Discrete Comput Geom 34:167-177 (2005)

DOI: $10.1007 / \mathrm{s} 00454-004-1155-\mathrm{x}$

\title{
Maximal Dimension of Unit Simplices
}

\author{
Christian Elsholtz ${ }^{1}$ and Walter Klotz ${ }^{2}$ \\ ${ }^{1}$ Department of Mathematics, Royal Holloway, University of London, \\ Egham, Surrey TW20 0EX, England \\ christian.elsholtz@rhul.ac.uk \\ ${ }^{2}$ Institut für Mathematik, Technische Universität Clausthal, \\ D-38678 Clausthal-Zellerfeld, Germany \\ klotz@math.tu-clausthal.de
}

\begin{abstract}
For an arbitrary field $\mathbb{F}$ the maximal number $\omega\left(\mathbb{F}^{n}\right)$ of points in $\mathbb{F}^{n}$ mutually distance 1 apart with respect to the standard inner product is investigated. If the characteristic $\operatorname{char}(\mathbb{F})$ is different from 2 , then the values of $\omega\left(\mathbb{F}^{n}\right)$ lie between $n-1$ and $n+2$. In particular, we answer completely for which $n$ a simplex of $q$ points with edge length 1 can be embedded in rational $n$-space. Our results imply for almost all even $n$ that $\omega\left(\mathbb{Q}^{n}\right)=n$ and for almost all odd $n$ that $\omega\left(\mathbb{Q}^{n}\right)=n-1$.
\end{abstract}

\section{Introduction}

\subsection{Motivation}

It is well known that the unit triangle cannot be embedded into $\mathbb{Q}^{2}$. In $\mathbb{Q}^{8}$ we easily find the regular unit simplex spanned by the following nine points:

$$
\left(\begin{array}{c}
\frac{1}{2} \\
\pm \frac{1}{2} \\
0 \\
0 \\
0 \\
0 \\
0 \\
0
\end{array}\right),\left(\begin{array}{c}
0 \\
0 \\
\frac{1}{2} \\
\pm \frac{1}{2} \\
0 \\
0 \\
0 \\
0
\end{array}\right),\left(\begin{array}{c}
0 \\
0 \\
0 \\
0 \\
\frac{1}{2} \\
\pm \frac{1}{2} \\
0 \\
0
\end{array}\right),\left(\begin{array}{c}
0 \\
0 \\
0 \\
0 \\
0 \\
0 \\
\frac{1}{2} \\
\pm \frac{1}{2}
\end{array}\right),\left(\begin{array}{c}
\frac{1}{2} \\
0 \\
\frac{1}{2} \\
0 \\
\frac{1}{2} \\
0 \\
\frac{1}{2} \\
0
\end{array}\right)
$$


In $\mathbb{R}^{n}$ we can obviously place exactly $n+1$ points defining a unit simplex. If we define $\omega\left(\mathbb{F}^{n}\right)$ to be the maximal number of points mutually at unit distance over the field $\mathbb{F}$, then $\omega\left(\mathbb{R}^{n}\right)=n+1$. (For a precise definition of the distance function see the next subsection.) On the other hand, $\omega\left(\mathbb{Q}^{n}\right)$ had previously only been determined up to the solution of a diophantine equation, see [4]. In this paper we solve this diophantine equation and thus determine $\omega\left(\mathbb{Q}^{n}\right)$ completely. It turns out that the prime factorizations of $n$ and $n+1$ are an important ingredient in the classification. A consequence of this result is that $\omega\left(\mathbb{Q}^{n}\right)=n$ for almost all even $n$ and $\omega\left(\mathbb{Q}^{n}\right)=n-1$ for almost all odd $n$.

The problem of determining $\omega\left(\mathbb{F}^{n}\right)$ is loosely connected to the problem of the chromatic number of $\mathbb{F}^{n}$. Let $\chi\left(\mathbb{F}^{n}\right)$ be the minimum number of colours needed to colour $\mathbb{F}^{n}$ so that no two points of the same colour are one unit apart. Even the determination of the chromatic number of the plane is a major outstanding problem. It is only known that $4 \leq \chi\left(\mathbb{R}^{2}\right) \leq 7$. For a survey see [10] and [11]. We may interpret $\omega\left(\mathbb{F}^{n}\right)$ as the clique number of the unit-distance graph $G_{1}\left(\mathbb{F}^{n}\right)$, which has vertex set $\mathbb{F}^{n}$ with vertices $x, y$ connected by an edge, if they are at unit distance, see [5]. Then $\omega\left(\mathbb{F}^{n}\right)$ is a lower bound for the chromatic number $\chi\left(G_{1}\left(\mathbb{F}^{n}\right)\right)=\chi\left(\mathbb{F}^{n}\right)$. For $\mathbb{F}=\mathbb{Q}$ some exact values are known: $\chi\left(\mathbb{Q}^{2}\right)=\chi\left(\mathbb{Q}^{3}\right)=2, \chi\left(\mathbb{Q}^{4}\right)=4[1],[12], \quad \chi\left(\mathbb{Q}^{5}\right) \geq 7$ [8], see also [13]. This compares very well with $\omega\left(\mathbb{Q}^{2}\right)=\omega\left(\mathbb{Q}^{3}\right)=2$ and $\omega\left(\mathbb{Q}^{4}\right)=\omega\left(\mathbb{Q}^{5}\right)=4$. While $\omega\left(\mathbb{Q}^{n}\right) \leq \omega\left(\mathbb{R}^{n}\right)=n+1$, the chromatic numbers $\chi\left(\mathbb{Q}^{n}\right)$ and $\chi\left(\mathbb{R}^{n}\right)$ increase exponentially, see [6] and [9].

\subsection{Results}

As many of our arguments hold for arbitrary fields, we start with a rather general setting. The most interesting applications are perhaps to subfields of $\mathbb{R}$.

Let $\mathbb{F}$ be an arbitrary field, let $x=\left(x_{i}\right) \in \mathbb{F}^{n}$ and let $y=\left(y_{i}\right) \in \mathbb{F}^{n}, n$ a positive integer. Define the quadratic distance of points $x$ and $y$ by the standard inner product:

$$
\Delta(x, y)=(x-y)^{2}=\sum_{i=1}^{n}\left(x_{i}-y_{i}\right)^{2} .
$$

By $\omega\left(\mathbb{F}^{n}\right)$ we denote the maximal number of points in $\mathbb{F}^{n}$, which mutually have quadratic distance 1.

We call the standard inner product of $\mathbb{F}^{n}$ nonisotropic if

$$
x \cdot x=0 \quad \Longleftrightarrow \quad x=0 \quad \text { for every } \quad x \in \mathbb{F}^{n} .
$$

Observe that, e.g., for $\operatorname{char}(\mathbb{F})=2$ and $n \geq 2$ the standard inner product is not nonisotropic, while it is nonisotropic for real fields.

In case of a nonisotropic inner product the next theorem completely determines $\omega\left(\mathbb{F}^{n}\right)$ up to the solution of a quadratic equation in the very last case, with values bounded by $n-1 \leq \omega\left(\mathbb{F}^{n}\right) \leq n+2$. For the proof we combine methods from design theory (Bruck-Ryser type arguments, see [3]) with those from linear algebra and number theory. 


\section{Theorem 1.}

(A) If $\operatorname{char}(\mathbb{F})=2$, then $\omega\left(\mathbb{F}^{n}\right)=2$ for every $n \geq 1$.

(B) If $\operatorname{char}(\mathbb{F}) \neq 2$ and $n$ is even, then the following statements hold true:

(1) If $\sqrt{n+1} \notin \mathbb{F}$, then $\omega\left(\mathbb{F}^{n}\right)=n$.

(2) Suppose that $\sqrt{n+1} \in \mathbb{F}$.

If $n=-2$ in $\mathbb{F}$, then $\omega\left(\mathbb{F}^{n}\right)=n+2$, otherwise $\omega\left(\mathbb{F}^{n}\right)=n+1$.

(C) If $\operatorname{char}(\mathbb{F}) \neq 2$ and $n$ is odd, then the following statements hold true:

(1) Suppose that $\sqrt{(n+1) / 2} \in \mathbb{F}$.

If $n=-2$ in $\mathbb{F}$, then $\omega\left(\mathbb{F}^{n}\right)=n+2$, otherwise $\omega\left(\mathbb{F}^{n}\right)=n+1$.

(2) Suppose that $\sqrt{(n+1) / 2} \notin \mathbb{F}$.

If the equation

$$
u^{2}+2(n-1) v^{2}=n
$$

has a solution with $u \in \mathbb{F}$ and $v \in \mathbb{F}$, then $\omega\left(\mathbb{F}^{n}\right)=n$.

If the standard inner product of $\mathbb{F}^{n}$ is nonisotropic and if equation (1) is unsolvable in $\mathbb{F}$, then $\omega\left(\mathbb{F}^{n}\right)=n-1$.

Theorem 1 immediately implies the following corollary:

Corollary 1. The smallest field $\mathbb{F}$ over $\mathbb{Q}$ such that $\omega\left(\mathbb{F}^{n}\right)=n+1$ for every positive integer $n$ is $\mathbb{F}=\mathbb{Q}[\sqrt{2}, \sqrt{3}, \sqrt{5}, \sqrt{7}, \ldots]$.

The next theorem describes the complete evaluation of $\omega\left(\mathbb{Q}^{n}\right)$.

\section{Theorem 2.}

(A) Let $n$ be even.

If $n+1$ is the square of an integer, then $\omega\left(\mathbb{Q}^{n}\right)=n+1$, otherwise $\omega\left(\mathbb{Q}^{n}\right)=n$.

(B) Let $n$ be odd.

(1) If $(n+1) / 2$ is the square of an integer, then $\omega\left(\mathbb{Q}^{n}\right)=n+1$.

(2) Suppose that $(n+1) / 2$ is not the square of an integer. Let $n=n_{1} n_{2}^{2}$ be the factorization of $n$ with a unique squarefree divisor $n_{1}$. If $n_{1}$ has no prime divisor $p \equiv \pm 3$ modulo 8 , then $\omega\left(\mathbb{Q}^{n}\right)=n$, and $\omega\left(\mathbb{Q}^{n}\right)=n-1$ otherwise.

Let $N_{\text {even }}(t)$ denote the number of even integers $n \leq t$ with $\omega\left(\mathbb{Q}^{n}\right) \neq n$ and $N_{\text {odd }}(t)$ the number of odd integers $n \leq t$ with $\omega\left(\mathbb{Q}^{n}\right) \neq n-1$. Evidently, part A of Theorem 2 implies $N_{\text {even }}(t) \sim \sqrt{t} / 2$. Landau's method [7, Sections 177-183] on estimating the number of positive integers $\leq t$ with specified prime factors only, allows us to conclude from part B of Theorem 2 that we have $N_{\text {odd }}(t) \sim c t /(\log t)^{1 / 2}$ for some positive constant $c$. So we may state the following consequence.

\section{Corollary 2.}

(1) $\omega\left(\mathbb{Q}^{n}\right)=n$ for almost all even integers $n$.

(2) $\omega\left(\mathbb{Q}^{n}\right)=n-1$ for almost all odd integers $n$. 
Theorem 2 and Corollary 2 have the following geometric interpretation. For those $n$ with $\omega\left(\mathbb{Q}^{n}\right)=n+1$ it is possible to rotate the regular $n$-dimensional simplex with edge length 1 in $\mathbb{R}^{n}$ so that all coordinates of the $n+1$ vertices become rational. For almost all dimensions $n$, however, this type of rotation does not exist and only a unit simplex with up to $n$ (for even $n$ ), respectively $n-1$ (for odd $n$ ), vertices can be embedded into $\mathbb{Q}^{n}$.

\section{Proof of Theorem 1}

First we settle the case char $(\mathbb{F})=2$ and establish the upper bounds. Let $x_{0}, \ldots, x_{m}$ be $m+1$ points in $\mathbb{F}^{n}$ mutually at quadratic distance 1 . We may suppose $x_{0}=0$. Then the other vectors $x_{j}, j \geq 1$, have unit length and for $i, j \geq 1, i \neq j$, we have

$$
\left(x_{i}-x_{j}\right)^{2}=x_{i}^{2}-2 x_{i} x_{j}+x_{j}^{2}=1, \quad 2 x_{i} x_{j}=1 .
$$

If $\operatorname{char}(\mathbb{F})=2$ and $m \geq 2$, then the last equation implies a contradiction. This proves part A.

From now on we assume $\operatorname{char}(\mathbb{F}) \neq 2$. Let the $(m \times n)$-matrix $A$ be formed by the rows $x_{1}, \ldots, x_{m}$. Then $A A^{T}$ is an $(m \times m)$-matrix with entries 1 in the main diagonal and $\frac{1}{2}$ in the other positions so that

$$
\operatorname{det} A A^{T}=(m+1) \frac{1}{2^{m}} .
$$

If $m \neq-1$ in $\mathbb{F}$, then $\operatorname{det} A A^{T} \neq 0$, so that

$$
\operatorname{rank} A A^{T}=m \leq \operatorname{rank} A \leq n .
$$

For $m=n+1$ and $n \neq-2$ in $\mathbb{F}$ inequality (3) leads to a contradiction, which implies

$$
\omega\left(\mathbb{F}^{n}\right) \leq n+1, \quad \text { if } \quad n \neq-2 \quad \text { in } \mathbb{F} .
$$

If $n=-2$ in $\mathbb{F}$, then a contradiction in (3) occurs for $m=n+2$, which shows

$$
\omega\left(\mathbb{F}^{n}\right) \leq n+2, \quad \text { if } \quad n=-2 \quad \text { in } \mathbb{F} .
$$

Let $m=n$. Then (2) may be written as

$$
n+1=2^{n}(\operatorname{det} A)^{2}
$$

If $n$ is even, then $n+1$ is the square of an element in $\mathbb{F}$, i.e. $\sqrt{n+1} \in \mathbb{F}$. If $n$ is odd, then

$$
(n+1) / 2=2^{n-1}(\operatorname{det} A)^{2}
$$

is the square of an element in $\mathbb{F}$, i.e. $\sqrt{(n+1) / 2} \in \mathbb{F}$. So we know

$$
\omega\left(\mathbb{F}^{n}\right) \leq n, \quad \text { if } \quad\left\{\begin{array}{l}
n \text { is even and } \sqrt{n+1} \notin \mathbb{F}, \\
n \text { is odd and } \sqrt{(n+1) / 2} \notin \mathbb{F} .
\end{array}\right.
$$

To establish lower bounds we start the constructive part of our proof. We define a system $\sigma$ of $n$ points $x^{(1)}, \ldots, x^{(n)}$ if $n$ is even, and of $n-1$ points $x^{(1)}, \ldots, x^{(n-1)}$ if $n$ 
is odd. If we take the vectors of $\sigma$ as the rows of a matrix, this matrix has the following shape:

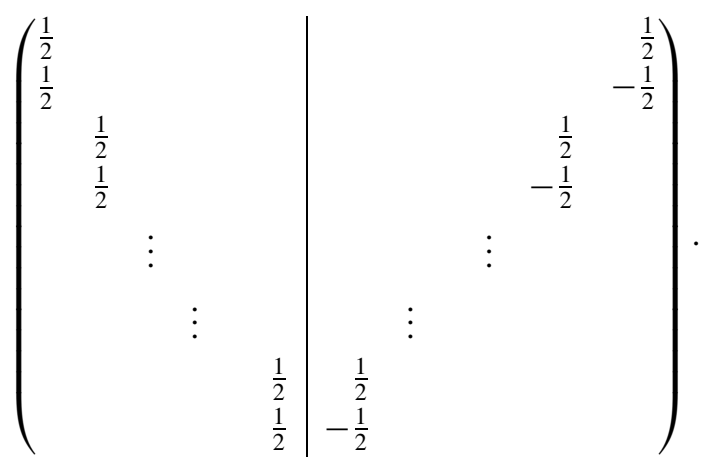

Positions left empty have to be filled with zeros. If $n$ is odd, a last column consisting of zeros only has to be added. Clearly, the points in $\sigma$ are mutually at distance 1 . Thus we have $\omega\left(\mathbb{F}^{n}\right) \geq n$ if $n$ is even and $\omega\left(\mathbb{F}^{n}\right) \geq n-1$ if $n$ is odd.

We try to extend the system $\sigma$ equidistantly by a point $y=\left(y_{1}, \ldots, y_{n}\right) \in \mathbb{F}^{n}$.

First we suppose that $n$ is even.

The additional point $y$ must have quadratic distance 1 to all points $x^{(1)}, \ldots, x^{(n)}$ of $\sigma$, which for $m=1, \ldots, n / 2$ implies

$$
\begin{gathered}
\Delta\left(y, x^{(2 m-1)}\right)=\left(y_{m}-\frac{1}{2}\right)^{2}+\left(y_{n-m+1}-\frac{1}{2}\right)^{2}+\sum_{i \neq m, n-m+1}\left(y_{i}\right)^{2}=1, \\
\Delta\left(y, x^{(2 m)}\right)=\left(y_{m}-\frac{1}{2}\right)^{2}+\left(y_{n-m+1}+\frac{1}{2}\right)^{2}+\sum_{i \neq m, n-m+1}\left(y_{i}\right)^{2}=1 .
\end{gathered}
$$

Taking the difference of these equations yields

$$
y_{n-m+1}=0 \quad \text { for } \quad m=1, \ldots, \frac{n}{2} .
$$

Now we have for $1 \leq m \leq n / 2-1$,

$$
\begin{gathered}
\Delta\left(y, x^{(2 m-1)}\right)=\left(y_{m}-\frac{1}{2}\right)^{2}+\frac{1}{4}+\sum_{i \neq m}\left(y_{i}\right)^{2}=1, \\
\Delta\left(y, x^{(2 m+1)}\right)=\left(y_{m+1}-\frac{1}{2}\right)^{2}+\frac{1}{4}+\sum_{i \neq m+1}\left(y_{i}\right)^{2}=1 .
\end{gathered}
$$

Again subtracting equations leads to

$$
y_{m+1}=y_{m} \quad \text { for } \quad m=1, \ldots, \frac{n}{2}-1 .
$$

Thus we see that $y$ must have the form

$$
y=y(s)=(\underbrace{s, \ldots, s}_{n / 2 \text { entries }}, \underbrace{0, \ldots, 0}_{n / 2 \text { entries }}), \quad s \in \mathbb{F} .
$$


Indeed, in this shape $y$ has the same distance to all points $x^{(j)}, j \leq n$.

$$
\begin{aligned}
\Delta\left(y, x^{(j)}\right)= & \left(s-\frac{1}{2}\right)^{2}+\left(\frac{n}{2}-1\right) s^{2}+\frac{1}{4}=1, \\
& \frac{n}{2} s^{2}-s-\frac{1}{2}=0 .
\end{aligned}
$$

If $n=0 \mathrm{in} \mathbb{F}$, then $\sigma$ can be extended equidistantly by $y\left(-\frac{1}{2}\right)$ to a system of $n+1$ points. According to (4) this is optimal, hence $\omega\left(\mathbb{F}^{n}\right)=n+1$. Notice that $\sqrt{n+1}=1 \in \mathbb{F}$ in this case, which corresponds to part $\mathrm{B}(2)$ of Theorem 1 .

If $n \neq 0$ in $\mathbb{F}$, then we solve (7) for $s$ :

$$
s=\frac{1}{n}(1 \pm \sqrt{n+1}) .
$$

An equidistant extension of $\sigma$ exists if and only if $\sqrt{n+1} \in \mathbb{F}$. If $\sqrt{n+1} \in \mathbb{F}$ and $n \neq-2$ in $\mathbb{F}$, then $\omega\left(\mathbb{F}^{n}\right)=n+1$ according to (4).

To finish the case of even $n$, suppose now $n=-2$ in $\mathbb{F}$ and $\sqrt{n+1}=\sqrt{-1} \in \mathbb{F}$. The only candidates for an equidistant extension of $\sigma$ are

$$
\begin{aligned}
& y^{(1)}=y\left(s_{1}\right) \quad \text { with } \quad s_{1}=\frac{1}{n}(1+\sqrt{n+1})=-\frac{1}{2}(1+\sqrt{-1}), \\
& y^{(2)}=y\left(s_{2}\right) \quad \text { with } \quad s_{2}=\frac{1}{n}(1-\sqrt{n+1})=-\frac{1}{2}(1-\sqrt{-1}) .
\end{aligned}
$$

The quadratic distance of $y^{(1)}$ and $y^{(2)}$ is

$$
\Delta\left(y^{(1)}, y^{(2)}\right)=\frac{n}{2}\left(s_{1}-s_{2}\right)^{2}=-(\sqrt{-1})^{2}=1,
$$

which means that in this case the system $\sigma$ can be extended equidistantly to a system of $n+2$ points. According to (5) no further extension is possible, $\omega\left(\mathbb{F}^{n}\right)=n+2$.

We now consider the case of odd $n$.

In this case the system $\sigma$ consists of $n-1$ points $x^{(1)}, \ldots, x^{(n-1)}$. Again we try to extend $\sigma$ equidistantly by a point $y=\left(y_{1}, \ldots, y_{n}\right)$. As above, the distance conditions

$$
\Delta\left(y, x^{(j)}\right)=1 \quad \text { for } \quad j=1, \ldots, n-1
$$

force $y$ to take the following form:

$$
y=y(s, v)=(\underbrace{s, \ldots, s}_{(n-1) / 2 \text { entries }}, \underbrace{0, \ldots, 0,}_{(n-1) / 2 \text { entries }} v), \quad s \in \mathbb{F}, \quad v \in \mathbb{F} .
$$

Indeed, in this shape $y$ has the same distance to all points $x^{(j)}, j<n$.

$$
\begin{gathered}
\Delta\left(y, x^{(j)}\right)=\left(s-\frac{1}{2}\right)^{2}+\left(\frac{n-1}{2}-1\right) s^{2}+\frac{1}{4}+v^{2}=1, \\
\frac{n-1}{2} s^{2}-s+v^{2}-\frac{1}{2}=0 .
\end{gathered}
$$


An equidistant extension of $\sigma$ exists if and only if (8) has a solution with $s \in \mathbb{F}$ and $v \in \mathbb{F}$.

If $n=1$ in $\mathbb{F}$, then (8) reduces to

$$
s=v^{2}-\frac{1}{2} .
$$

For $v= \pm \frac{1}{2}, s=-\frac{1}{4}$ we get two points, which extend $\sigma$ equidistantly to a system of $n+1$ points. If $n \neq-2$ in $\mathbb{F}$, this leads to $\omega\left(\mathbb{F}^{n}\right)=n+1$ according to (4). Notice that in this case $\sqrt{(n+1) / 2}=1 \in \mathbb{F}$, which supports part C(1) of Theorem 1. If simultaneously $n=1$ and $n=-2$ in $\mathbb{F}$, then $\operatorname{char}(\mathbb{F})=3$. In this case we can find three points for an equidistant extension of $\sigma$ :

$$
y^{(1)}=y\left(\frac{1}{2}, 1\right), \quad y^{(2)}=y\left(\frac{1}{2},-1\right), \quad y^{(3)}=y\left(-\frac{1}{2}, 0\right) .
$$

According to (5) no further extension is possible. So in this case $\omega\left(\mathbb{F}^{n}\right)=n+2$, which again corresponds to part $\mathrm{C}(1)$ of Theorem 1.

Suppose now $n \neq 1$ in $\mathbb{F}$. We solve (8) for $s$ :

$$
s=\frac{1}{n-1}\left(1 \pm \sqrt{1-2(n-1)\left(v^{2}-\frac{1}{2}\right)}\right) .
$$

There is an equidistant extension of $\sigma$ if and only if the equation

$$
\begin{gathered}
1-2(n-1)\left(v^{2}-\frac{1}{2}\right)=u^{2} \quad \text { or, equivalently, } \\
u^{2}+2(n-1) v^{2}=n
\end{gathered}
$$

has a solution with $u \in \mathbb{F}$ and $v \in \mathbb{F}$. We try to find a solution of (10) for $v= \pm \frac{1}{2}$.

$$
u^{2}+2(n-1) \frac{1}{4}=n, \quad u= \pm \sqrt{(n+1) / 2} .
$$

If $\sqrt{(n+1) / 2} \in \mathbb{F}$, then we determine $s \in \mathbb{F}$ for $v= \pm \frac{1}{2}$ by (9). The points $y^{(1)}=$ $y\left(s, \frac{1}{2}\right), y^{(2)}=y\left(s,-\frac{1}{2}\right)$ extend $\sigma$ equidistantly to a system of $n+1$ points. If $n \neq-2$ in $\mathbb{F}$, then by (4) we conclude $\omega\left(\mathbb{F}^{n}\right)=n+1$. If $n=-2$ in $\mathbb{F}$, then we take a closer look at the common element $s$ in $y^{(1)}$ and $y^{(2)}$, which we get from (9):

$$
s=-\frac{1}{3}\left(1+\sqrt{-\frac{1}{2}}\right)=-\frac{1}{3}\left(1+\frac{1}{2} \sqrt{-2}\right) .
$$

Notice that $\operatorname{char}(\mathbb{F}) \neq 3$ and $\sqrt{-2} \in \mathbb{F}$ in this case. Now (10) has a further solution in $\mathbb{F}: v=0, u=\sqrt{-2}$. For $v=0$ we determine $s_{0}$ by (9):

$$
s_{0}=-\frac{1}{3}(1-\sqrt{-2}) \text {. }
$$

It is easily checked that $\sigma$ can be extended equidistantly by the following three points:

$$
y^{(1)}=y\left(s, \frac{1}{2}\right), y^{(2)}=y\left(s,-\frac{1}{2}\right), \quad y^{(3)}=y\left(s_{0}, 0\right) .
$$

This shows $\omega\left(\mathbb{F}^{n}\right)=n+2$ in this case and completes the proof of part C(1) in Theorem 1 . 
To finish the proof of part $\mathrm{C}(2)$ of Theorem 1 we may assume that $n$ is odd and $\sqrt{(n+1) / 2} \notin \mathbb{F}$. In this case the possible values of $\omega\left(\mathbb{F}^{n}\right)$ are $n-1$ and $n$. We show that it is no loss of generality, if we start with the special points studied above. For the points $x^{(1)}, \ldots, x^{(n-1)}$ of $\sigma$ we set

$$
w^{(j)}=x^{(j+1)}-x^{(1)}, \quad j=1, \ldots, n-2 .
$$

Then we have $\left(w^{(j)}\right)^{2}=1$ and $w^{(j)} w^{(k)}=\frac{1}{2}$ for $j \neq k$. If there is any system of $n$ points in $\mathbb{F}^{n}$ mutually at quadratic distance 1 , then we could analogously find vectors $z^{(1)}, \ldots, z^{(n-1)}$ satisfying $\left(z^{(j)}\right)^{2}=1$ and $z^{(j)} z^{(k)}=\frac{1}{2}$ for $j \neq k$. Now a theorem from linear algebra (Proposition 2 of the Appendix) guarantees the existence of an isometry $f$ of $\mathbb{F}^{n}$ with

$$
f\left(z^{(j)}\right)=w^{(j)} \quad \text { for } \quad j=1, \ldots, n-2 .
$$

For this proposition we need $\operatorname{char}(\mathbb{F}) \neq 2$ and the assumption that the standard inner product of $\mathbb{F}^{n}$ is nonisotropic. Now

$$
y=x^{(1)}+f\left(z^{(n-1)}\right)
$$

would have quadratic distance 1 to every point $x^{(j)}, j<n$. This means that $\omega\left(\mathbb{F}^{n}\right)=n$ if and only if the system $\sigma$ of points $x^{(1)}, \ldots, x^{(n-1)}$ has an equidistant extension by an additional point $y$. We have already shown that such an extension exists if and only if (10) is solvable in $\mathbb{F}$. This completes the proof of Theorem 1.

\section{Proof of Theorem 2}

Parts A and $\mathrm{B}(1)$ immediately follow from Theorem 1. Let $n$ be odd and let $(n+1) / 2$ not be the square of an integer. From Theorem 1 part $\mathrm{C}(2)$ we conclude $\omega\left(\mathbb{Q}^{n}\right)=n$, if the equation

$$
u^{2}+2(n-1) v^{2}=n
$$

has a solution with $u, v \in \mathbb{Q}$ and $\omega\left(\mathbb{Q}^{n}\right)=n-1$, if this equation is unsolvable in $\mathbb{Q}$. We may set

$$
u=\frac{z}{x}, \quad v=\frac{y}{x} \quad \text { with integers } \quad x, y, z, x \neq 0,
$$

and so arrive at the diophantine equation of Chilakamarri [4]:

$$
n x^{2}-2(n-1) y^{2}=z^{2} .
$$

If this equation has a solution in integers $x, y, z$ with $x \neq 0$, then $\omega\left(\mathbb{Q}^{n}\right)=n$, otherwise $\omega\left(\mathbb{Q}^{n}\right)=n-1$. So the proof of Theorem 2 will be accomplished, if the following proposition is shown to be true.

Proposition 1. Let $n$ be an integer $\neq 0$ and let $n=n_{1} n_{2}^{2}$ be the factorization of $n$ with unique squarefree divisor $n_{1}$. The diophantine equation

$$
n x^{2}-2(n-1) y^{2}=z^{2}
$$


has a solution in integers $x, y, z, x \neq 0$, if and only if $n_{1}$ has no prime divisor $p \equiv \pm 3$ modulo 8.

To prove Proposition 1 we take advantage of the following result from number theory.

Lemma 1. Let $n$ be an integer $\neq 0$ and let $n=n_{1} n_{2}^{2}$ be the factorization of $n$ with unique squarefree divisor $n_{1}$. The diophantine equation

$$
x^{2}-2 y^{2}=n
$$

has a solution in integers $x, y, z, x \neq 0$, if and only if $n_{1}$ has no prime divisor $p \equiv \pm 3$ modulo 8.

The proof of Lemma 1 is analogous to the well-known classification of those integers that can be written as the sum of two squares. A full proof of Lemma 1 can be found as Theorem 35.3 in [2].

Proof of Proposition 1. Equation (11) can be transformed to

$$
n\left(x^{2}-2 y^{2}\right)=z^{2}-2 y^{2} .
$$

Suppose $x, y, z, x \neq 0$, are integers satisfying (11). As $n \neq 0, x \neq 0$ and as $\sqrt{2}$ is irrational, the integers $x^{2}-2 y^{2}, z^{2}-2 y^{2}$ are nonzero and according to Lemma 1 have prime divisors $p \equiv \pm 3$ modulo 8 only with even multiplicity $\geq 0$. Equation (12) implies that the same must be true for $n$.

Now let $n$ have prime divisors $p \equiv \pm 3$ modulo 8 only with even multiplicity $\geq 0$. By Lemma 1 we can find integers $a, b$ with $a^{2}-2 b^{2}=n$. We may assume that $a \neq 1$. It is easily checked that

$$
x=a-1, \quad y=b, \quad z=a(a-1)-2 b^{2}
$$

solves (11).

\section{Problems and Remarks}

The above investigation can no doubt be extended in various directions.

1. Find a complete evaluation of $\omega\left(\mathbb{F}^{n}\right)$ for special fields such as finite fields or $\mathbb{Q}[\sqrt{p}], p$ a prime number.

2. Try to extend Corollary 2 to finite extensions of $\mathbb{Q}$.

3. If $\mathbb{F}$ is not a real field, then another distance function may be more appropriate.

\section{Acknowledgement}

We thank the unknown referees for their helpful comments. 


\section{Appendix}

To make this paper more self-contained we outline the result from linear algebra used for the proof of the last case in Theorem 1.

Assume $\operatorname{char}(\mathbb{F}) \neq 2$ and let the standard inner product of $\mathbb{F}^{n}$ be nonisotropic. An isometry $f$ of $\mathbb{F}^{n}$ is a length preserving endomorphism of $\mathbb{F}^{n}$.

$f(x) \cdot f(x)=x \cdot x \quad$ for every $\quad x \in \mathbb{F}^{n} \Longleftrightarrow f(x) \cdot f(y)=x \cdot y$ for all $x, y \in \mathbb{F}^{n}$.

For $v \in \mathbb{F}^{n}, v \neq 0$ let $H(v)=\left\{x \in \mathbb{F}^{n}: v \cdot x=0\right\}$ be the hyperplane with normal vector $v$. Every $x \in \mathbb{F}^{n}$ can be uniquely written as

$$
x=x_{H}+\lambda \nu, \quad x_{H} \in H(\nu), \quad \lambda \in \mathbb{F} .
$$

The reflection $S_{v}$ at the hyperplane $H(v)$ is defined by $S_{v}(x)=x_{H}-\lambda \nu$. Clearly, $S_{v}$ is an isometry.

Proposition 2. Assume that the standard inner product of $\mathbb{F}^{n}$ is nonisotropic and $\operatorname{char}(\mathbb{F}) \neq 2$. Let $a_{1}, \ldots, a_{m}, b_{1}, \ldots, b_{m}$ be vectors in $\mathbb{F}^{n}$ satisfying

$$
a_{i} \cdot a_{j}=b_{i} \cdot b_{j} \quad \text { for all } \quad i, j=1, \ldots, m .
$$

Then there is an isometry $f$ of $\mathbb{F}^{n}$ with $f\left(a_{i}\right)=b_{i}$ for every $i=1, \ldots, m$. Either $f=\mathrm{id}$ or $f$ is the product of at most $m$ hyperplane reflections.

Proof. We may start the induction on $m$ formally with $m=0$ and $f=$ id. For the inductive step suppose that $m \geq 1$ and that the assertion is true for $m-1$. If $a_{1}, \ldots, a_{m}, b_{1}, \ldots, b_{m}$ satisfy the conditions of the proposition, then there is an isometry $\varphi$ with $\varphi\left(a_{i}\right)=b_{i}$ for $i=1, \ldots, m-1$, where $\varphi=$ id $\operatorname{or} \varphi$ is the product of at most $m-1$ hyperplane reflections. If $\varphi\left(a_{m}\right)=b_{m}$, then we may take $f=\varphi$. Let $v=\varphi\left(a_{m}\right)-b_{m} \neq 0, f=S_{\nu} \varphi$. For $i<m$ we have

$$
\nu \cdot \varphi\left(a_{i}\right)=\varphi\left(a_{m}\right) \cdot \varphi\left(a_{i}\right)-b_{m} \cdot \varphi\left(a_{i}\right)=a_{m} \cdot a_{i}-b_{m} \cdot b_{i}=0 .
$$

Therefore $\varphi\left(a_{i}\right) \in H(v)$ and

$$
f\left(a_{i}\right)=S_{v}\left(\varphi\left(a_{i}\right)\right)=\varphi\left(a_{i}\right)=b_{i} \quad \text { for every } \quad i, 1 \leq i<m .
$$

Now $\left(\varphi\left(a_{m}\right)\right)^{2}-b_{m}^{2}=a_{m}^{2}-b_{m}^{2}=0$ implies $\left(\varphi\left(a_{m}\right)-b_{m}\right) \cdot\left(\varphi\left(a_{m}\right)+b_{m}\right)=0$. From $v=\varphi\left(a_{m}\right)-b_{m}$ and $\left(\varphi\left(a_{m}\right)+b_{m}\right) \in H(v)$ we conclude

$$
\left.\begin{array}{l}
S_{v}\left(\varphi\left(a_{m}\right)+b_{m}\right)=\varphi\left(a_{m}\right)+b_{m} \\
S_{v}\left(\varphi\left(a_{m}\right)-b_{m}\right)=-\varphi\left(a_{m}\right)+b_{m}
\end{array}\right\} \quad \Longrightarrow \quad S_{v}\left(\varphi\left(a_{m}\right)\right)=f\left(a_{m}\right)=b_{m} .
$$

\section{References}

1. M. Benda and M. Perles, Colorings of metric spaces, Geombinatorics 9(3) (2000), 113-126.

2. E.D. Bolker, Elementary Number Theory, Benjamin, New York, 1970. 
3. R.H. Bruck and H.J. Ryser, The nonexistence of certain finite projective planes, Canad. J. Math. 1 (1949), 88-93.

4. K.B. Chilakamarri, Unit-distance graphs in rational $n$-spaces, Discrete Math. 69 (1988), 213-218.

5. K.B. Chilakamarri, The unit-distance graph problem: a brief survey and some new results, Bull. Inst. Combin. Appl. 8 (1993), 39-60.

6. P. Frankl and R.M. Wilson, Intersection theorems with geometric consequences. Combinatorica 1(4) (1981), 357-368.

7. E. Landau, Handbuch der Lehre von der Verteilung der Primzahlen, Chelsea (reprint), New York, 1953.

8. M. Mann, A new bound for the chromatic number of the rational five-space, Geombinatorics 11(2) (2001), 49-54.

9. A.M. Raigorodskij, Borsuk's problem and the chromatic numbers of some metric spaces, Russian Math. Surveys 56 (2001), 103-139.

10. S. Shelah and A. Soifer, Axiom of choice and chromatic number of the plane, J. Combin. Theory Ser. A 103 (2003), 387-391.

11. A. Soifer, Chromatic number of the plane: its past and future, Congr. Numer. 160 (2003), 69-82.

12. J. Zaks, On four-colourings of the rational four-space, Aequationes Math. 37 (1989), 259-266.

13. J. Zaks, On the chromatic number of some rational spaces. Ars Combin. 33 (1992), 253-256.

Received December 4, 2003, and in revised form August 8, 2004. Online publication January 14, 2005. 